Copyright (C 2014 IEEE. Personal use of this material is permitted. Permission from IEEE must be obtained for all other uses, in any current or future media, including reprinting/republishing this material for advertising or promotional purposes, creating new collective works, for resale or redistribution to servers or lists, or reuse of any copyrighted component of this work in other works. 


\title{
Interconnected Microgrids via Back-to-Back Converters for Dynamic Frequency Support
}

\author{
Julius Susanto, Farhad Shahnia, Arindam Ghosh and Sumehda Rajakaruna \\ Department of Electrical and Computer Engineering \\ Curtin University \\ Perth, Australia \\ julius.susanto@student.curtin.edu.au
}

\begin{abstract}
This paper presents the back-to-back interconnection of two autonomous microgrids. A back-to-back converter topology, referred to as an interchange converter, is used to provide dynamic frequency support for each autonomous microgrid. The proposed interchange converter facilitates active power exchange between the two microgrids when one cluster does not have sufficient generation capacity to supply its local load demand. During an overloading in the first microgrid, the output power of the energy resources within the other microgrid increases to supply the loads in the first microgrid. Hence, the frequency in both microgrids is kept within the acceptable range. Several study cases are simulated in DIgSILENT PowerFactory software to investigate and verify the successful dynamic frequency support by the help of the interchange converter.
\end{abstract}

Keywords-Interconnected microgrids, Back-to-back converter, Distributed energy resource (DER), Frequency

\section{INTRODUCTION}

Microgrid (MG) is a cluster of loads and generators that can act as a single controllable unit. The generators are considered as distributed energy resources (DERs) which are usually in the form of power electronic interfaced DERs, such as photovoltaic cells, wind turbines, micro-hydro turbines, battery storage and fuel cells systems or inertial DERs, such as induction/synchronous generators [1][1].

Microgrids can work in grid-connected or islanded (autonomous) modes. In grid-connected mode, the network voltage and frequency is dictated by the grid and the DERs generate the rated power. However, in autonomous mode, the DERs not only supply the active/reactive power demand of the loads within the MG, they are responsible for holding the voltage and frequency of the MG within acceptable ranges [2].

Recently, MGs have been considered as a possible power supply system for remote areas where external electricity grid is not available. This is mainly driven by the rising energy costs, advancements in DERs technology as well as the decreasing capital costs for installation of the DERs [3][3-4]. In such conditions, the MGs will operate in autonomous mode. It is to be noted that, the electric power network of one area can be composed of several neighbouring MGs, distributed within a small geographical area.

One of the key issues with such autonomous MGs is the possibility of observing that the generation/storage capacity is not sufficient to supply the load demand. This can happen as a result of overloading or a generation shortfall, such as the fluc- tuations in the output power of wind or photovoltaic-based DERs. In such cases, the existing under-frequency and undervoltage protection relays need to shed a portion of the loads to hold the voltage and frequency within acceptable ranges and prevent a network blackout [5].

However, it might so happen that in the considered geographical area, one of the autonomous MGs has a sufficient generation/storage capability when the other neighbouring autonomous MG has a deficiency in power. In [6], it is proposed that neighbouring MGs can be interconnected together to support each other under such scenarios. In [6], it is considered that the neighbouring autonomous MGs are connected together through a medium voltage backbone network. Another alternative is connecting the neighbouring MGs through a back-to-back converter-based system, as shown in Fig. 1(a). The main advantage of this interconnection is that each $\mathrm{MG}$ can operate at any voltage and frequency while they exchange active/reactive power through the back-to-back converters. Depending on the distance that the two neighbouring MGs are situated, the DC link can have a length of zero to a few kilometres.

This paper investigates how two neighbouring autonomous MGs can be interconnected via a back-to-back converter arrangement to provide frequency support through active power exchange (when required), while keeping the two MGs largely decoupled during normal operation.

The MG structure and its hierarchical control system are discussed in Section II. In Section III, the back-to-back interchange converter is introduced and the proposed control strategy is discussed. DIgSILENT PowerFactory based simulations are presented in Section IV which demonstrate the dynamic performance of the two interconnected MGs and the interchange converter.

\section{MICROGRID STRUCTURE AND CONTROL}

The main objective of this paper is to consider two neighbouring MG systems, situated at a close distance within a geographical area. Hence, the typical MG systems used as the power supply unit of the remote areas are focused in the paper. Thereby, two similar hybrid MG systems are assumed. Each hybrid MG system consists of two converter-interfaced DERs and one inertial DER. The DERs are assumed to have Voltage Source Converters (VSC) while the inertial DER is assumed to be a diesel generator. Fig. 1(b) shows the structure of the considered each hybrid MG system. 
The diesel generator set is modelled dynamically with the simple Siemens DEGOV diesel governor model [7] and the IEEE AC5A exciter model [8]. The diesel generator set is as shown in Fig. 1(c).

Each converter is composed of a three-phase, three-leg VSC structure as shown in Fig. 1(d).

Each MG has a hierarchical control system to maintain the frequency and voltage deviations within an acceptable range while the DERs supply the required active/reactive power demand of the local loads. The hierarchical control system for each MG is based on a three-level control system, similar to that used in large power systems, but is adapted for the MG [9-10]. These three levels are:

1. Primary control: This controller consists of two loopsinner loop and outer loop. The inner loop is responsible for regulating the output current and voltage of each converter-based DER. The outer-loop is responsible for defining the set-points for the inner-loop using the droop control and based on the set-points defined from the secondary controller.

For inertial DERs (i.e. diesel generator), the primary controller consists of a mechanically-actuated diesel engine governor with droop control.

2. Secondary control: This controller is responsible to define the proper set-points for the primary controllers of each DER such that the frequency and voltage within the MG is kept within the acceptable ranges.

3. Tertiary control: This controller facilitates the power exchange through the interchange converter between the two MGs. This control system is discussed in more detail in Section III.

In the control scheme proposed in this paper, a synchronous based diesel generator provides the reference voltage and frequency.

\section{A. Primary Control}

For converter-based DERs, a vector current control approach in the Direct-Quadrature (dq) reference frame is used for the inner control loop of each converter. The three-phase currents at the output of the converter $\left(I_{a b c}\right)$ are transferred to their equivalent direct-axis current $\left(I_{d}\right)$ and quadrature-axis current $\left(I_{q}\right)$. These two currents are regulated to their reference values (i.e. $I_{d, r e f}$ and $I_{q, r e f}$ ) by the help of Proportional-Integral (PI) controllers.

The outputs of the inner current controller are the modulation indices in the dq reference frame, which are then converted to the stationary abc frame and fed into the converter PWM modulator block [11]. This control block diagram is shown in Fig. 2(a).

Voltage and frequency droop control serves as the primary control strategy for each DER (synchronous and converterbased alike). The primary control structure is based on the standard droop equations as

$$
\begin{aligned}
& f=f_{\text {ref }}-m\left(P-P_{\text {ref }}\right) \\
& V=V_{\text {ref }}-n\left(Q-Q_{\text {ref }}\right)
\end{aligned}
$$

where $f$ and $V$ are respectively the frequency and voltage of (a)

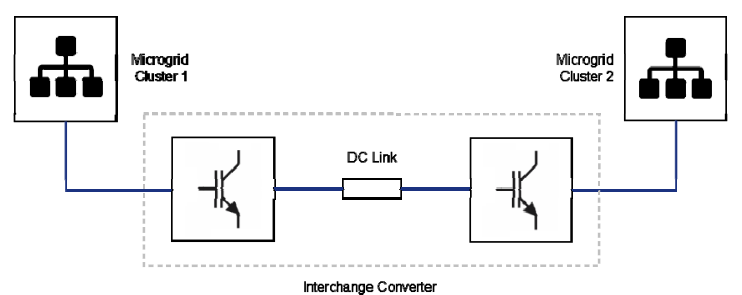

(b)

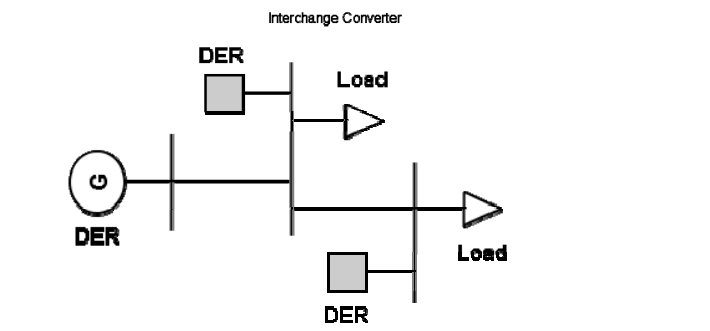

(c)

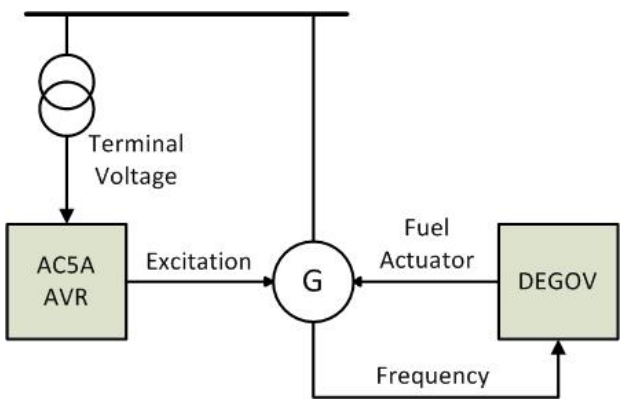

(d)

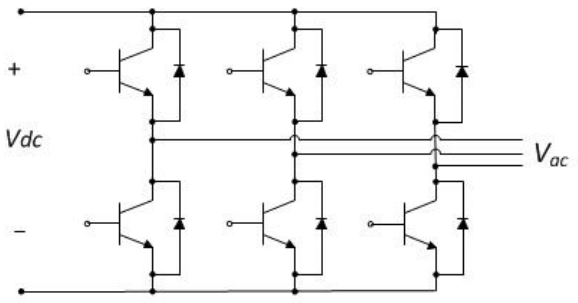

Fig. 1. Schematic diagram of power supply system for remote areas: (a) Two neighbouring autonomous MGs interconnected via interchange (back-toback) converter, (b) MG system consisting of two converter-interfaced DERs, one diesel generator and one local load, (c) Diesel genertor set, (d) VSC system of the converter-interfaced DERs.

the MG and $f_{\text {ref }}$ and $V_{\text {ref }}$ are the references for these parameters, $P$ and $Q$ are the average three-phase active and reactive power output of the DER and $P_{r e f}$ and $Q_{r e f}$ are the references for these parameters, and $m$ and $n$ are the frequency and voltage droop coefficients. It is to be noted that the reference values and the droop coefficients are defined by the secondary controller to provide a desired power ratio among the DERs as well as to hold the voltage and frequency within the acceptable ranges.

The droop coefficients are defined by the secondary controller

$$
\begin{aligned}
& m=\Delta f / P_{\max } \\
& n=\Delta V / 2 Q_{\max }
\end{aligned}
$$

where $\Delta f$ and $\Delta V$ are the maximum frequency and voltage deviations allowed and $P_{\max }$ and $Q_{\max }$ are the maximum active and reactive power output of the DER.

For converter-based DERs, the control of $P$ and $Q$ are achieved through the adjustment of inner control loop reference set-points (i.e. $I_{d, r e f}$ and $I_{q, r e f}$ ) from 


$$
\begin{aligned}
& I_{d, r e f}=P_{\text {ref }}+\left(f_{\text {ref }}-f\right) / m \\
& I_{q, \text { ref }}=Q_{\text {ref }}+\left(V_{\text {ref }}-V\right) / n
\end{aligned}
$$

\section{B. Secondary Control}

The secondary controller adjusts the active and reactive power reference signals $P_{r e f}$ and $Q_{\text {ref }}$ in order to regulate frequency and voltage to nominal values. Secondary control is intended to operate after primary control action has taken place, and so the time steps for the secondary controller are relatively larger compared to that of the primary controller (e.g. $3 \mathrm{~s}$ vs $10 \mathrm{~ms}$ ).

In the secondary controller, new active and reactive power set-points are calculated at discrete time intervals (e.g. every 3s) from

$$
\begin{aligned}
& P_{\text {ref }}=P+m\left(f-f_{\text {ref }}\right) \\
& Q_{\text {ref }}=Q+n\left(V-V_{\text {ref }}\right)
\end{aligned}
$$

\section{INTERCHANGE CONVERTER}

A back-to-back converter arrangement is proposed as a means to interconnect two neighbouring MGs. This converter will provide frequency support for the two MGs in the case of power deficiency in one of the MGs while the other MG has enough generation/storage capability. In this paper, the tertiary control is applied to control the dynamic performance of the interchange converter to support two interconnected MGs. Each converter is composed of a three-phase, three-leg VSC structure as shown in Fig. 2(d).

Each converter in the proposed back-to-back converter arrangement has an individual controller that is configured to regulate the voltage of the DC link and provide frequency support to both MGs. The control block diagram for each of these converters is shown in Fig. 3(a).

Each controller participates in controlling both the DC link voltage and the frequency of the MG. For this, an error signal $(\zeta)$ is calculated as

$$
\zeta=k_{d c}\left(V_{d c, r e f}-V_{d c}\right)+k_{f}\left(f_{\text {ref }}-f\right)
$$

where $V_{d c}$ is the DC voltage at the terminals of the converter and $V_{d c, r e f}$ is its reference value and $k_{f}$ and $k_{d c}$ are the controller coefficients. This error signal is then passed through a PI controller to generate $I_{d, r e f}$ for the converter. This is referred to as the active power controller module and is shown schematically in Fig. 3(b).

In this paper, the current controller for the converter is a based on a PI controller, similar to the inner-loop control of the DER converters.

\section{Study CASES AND SimUlation Results}

The remote area power supply unit shown in Fig. 1(a) is modelled. Two similar hybrid MGs are considered as shown in Fig. 1(b). Each MG is composed of one $6 \mathrm{kVA}$ diesel generator, as modelled in Fig. 1(c) and two $10 \mathrm{~kW}$ three-phase DERs, as modelled in Fig. 1(d). The MG is assumed to be a low voltage $(415 \mathrm{~V})$ network with two constant power (PQ) loads connected. A combined engine, flywheel and alternator inertia constant of $H=1 \mathrm{~s}$ is used for the diesel generator set.

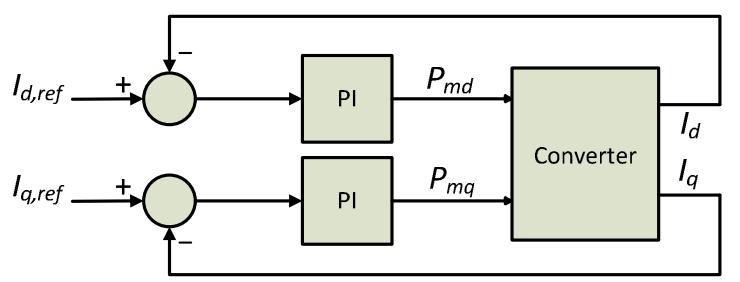

Fig. 2. Control block diagram of the MG: (a) Primary Control level.

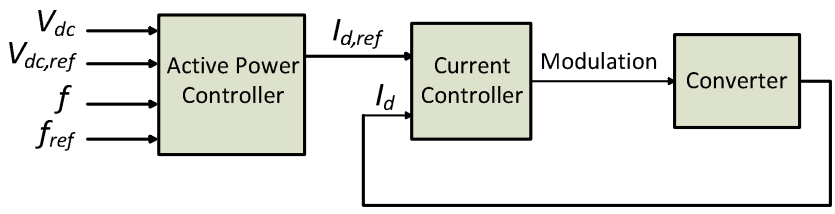

(a)

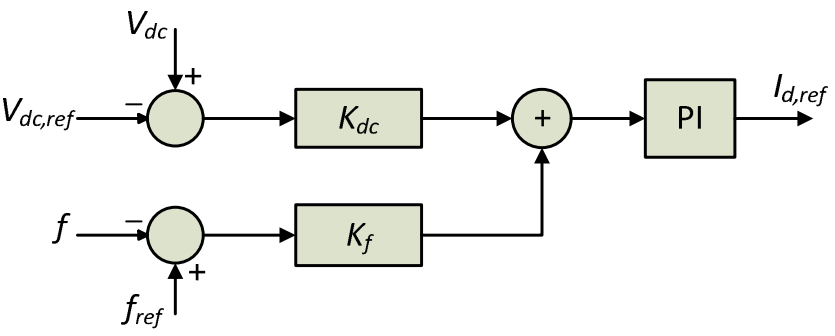

(b)

Fig. 3. (a) VSC structure of each converter in the interchange (back-to-back) converter, (b) Control block diagram of the interchnage converter, (c) Active power controller module of the interchnage converter.

At steady state, each MG operates autonomously, with the DERs supplying the loads in the MG. The primary controllers in each DER are set to $5 \%$ voltage and frequency droop, while the secondary controllers are set to recalculate the voltage and frequency reference values at discrete intervals of 3 seconds. The interchange converter consists of two $30 \mathrm{kVA}$ converters, as shown in Fig. 3(a) with $3 \%$ series coupling inductances connected together via a line with total resistance of $1.2 \Omega$.

The operation of the proposed interconnected microgrid system is investigated through simulations in DIgSILENT PowerFactory v15.1 software.

\section{A. Case 1: MG Normal Operation}

During normal operation, the local DERs in each MG supply the active and reactive power demand of the local loads. In Case 1, a modest load step is simulated in MG-1 such that the total load after the event is still within the combined capacity of the DER units.

To investigate the performance of the interconnected $\mathrm{MG}$ system under such a condition, let us assume that in the steady state, each MG has a load demand of $5 \mathrm{~kW}$ at pf $=0.85$ lagging. It is also assumed that, based on the secondary controller, this power is shared with a ratio of $60 / 40 \%$ between the two converter-interfaced DERs while the diesel generator is assumed to be running at no-load condition. The MG frequency and voltage magnitude are respectively $50 \mathrm{~Hz}$ and $1.001 \mathrm{pu}$. The results of the simulation are shown in Fig. 4.

At $t=1 \mathrm{~s}$, a $3 \mathrm{~kW}$ load (with $\mathrm{pf}=0.85$ lagging) is added to MG-1 (Fig. 4a). It can be seen that this additional demand is picked up by the two converter-interfaced DERs (i.e. con- 
verter-A, B) from both active power (Fig. $4 \mathrm{~b}$ ) and reactive power (Fig. 4c) points of view, based on the assigned $60 / 40 \%$ ratio. It is to be noted that although the diesel generator contributes during the transient period, it does not contribute to this additional load when the system reaches the steady-state condition at $t=12.5 \mathrm{~s}$ (Fig. 4b-c).

The MG frequency is restored to $50 \mathrm{~Hz}$ when the system reaches the steady-state condition (Fig. 4d) while the voltage rms restores back to $1 \mathrm{pu}$ at this time (Fig. 4e).

Due to the slight changes in the DC link voltage (Fig. 4f), the step load applied to MG-1 causes some oscillations in the system of MG-2; however, these oscillations are damped very quickly. At steady-state, it can be seen that the additional load demand in MG-1 is adequately supplied by the local DERs (converter-A and B), and there is very limited contribution from converter-C and D in MG-2 (Fig. 4g-h). Hence, the frequency and voltage rms of MG-2 restore back to their initial values (Fig. $4 \mathrm{i}-\mathrm{j}$ ). The presence of the interchange converter causes the frequency disturbance in MG-1 to ripple through to MG-2, but the effects are very small (i.e. $0.3 \%$ ). The active and reactive power exchange through the interchange converter is negligible at this condition (Fig. 4k-1).

\section{B. Case 2: MG Overload Operation without Interchange Converter}

When one of the MGs is overloaded, the total load demand in a MG exceeds the generation/storage capacity of the DERs. In Case 2, the performance of the MG is first examined without the influence of the interchange converter.

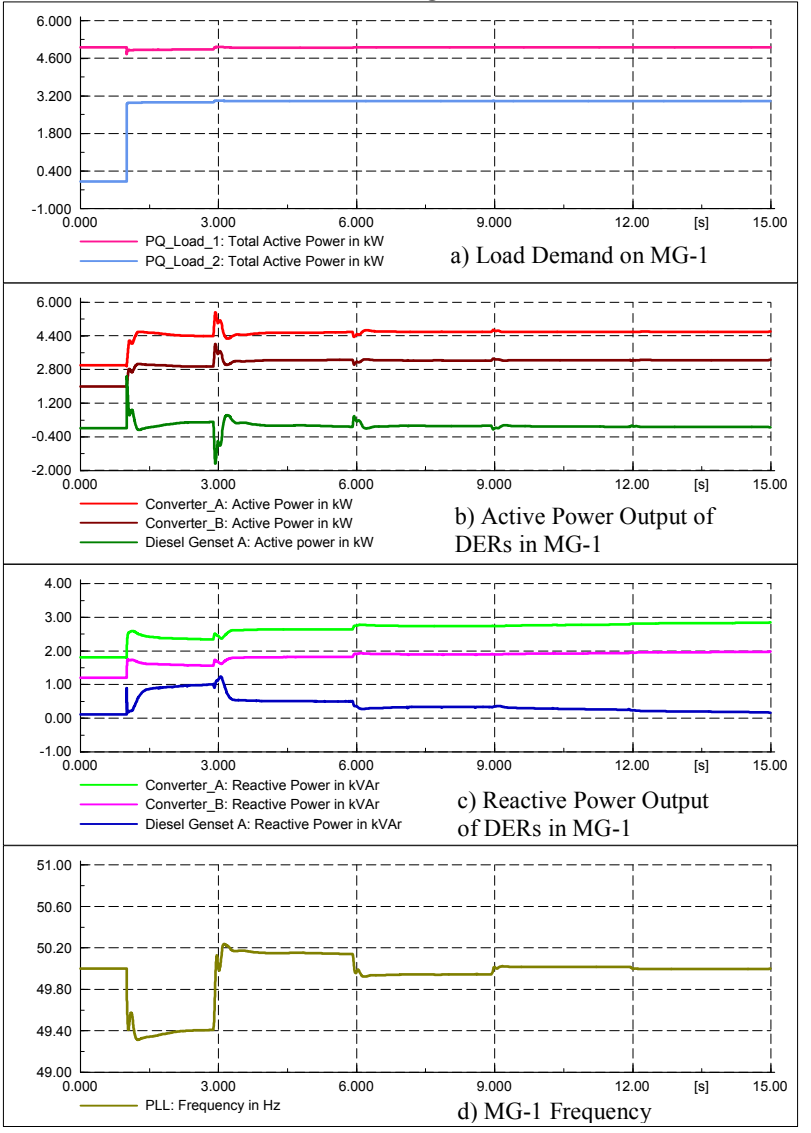

Fig. 4. Case 1 simulation results.

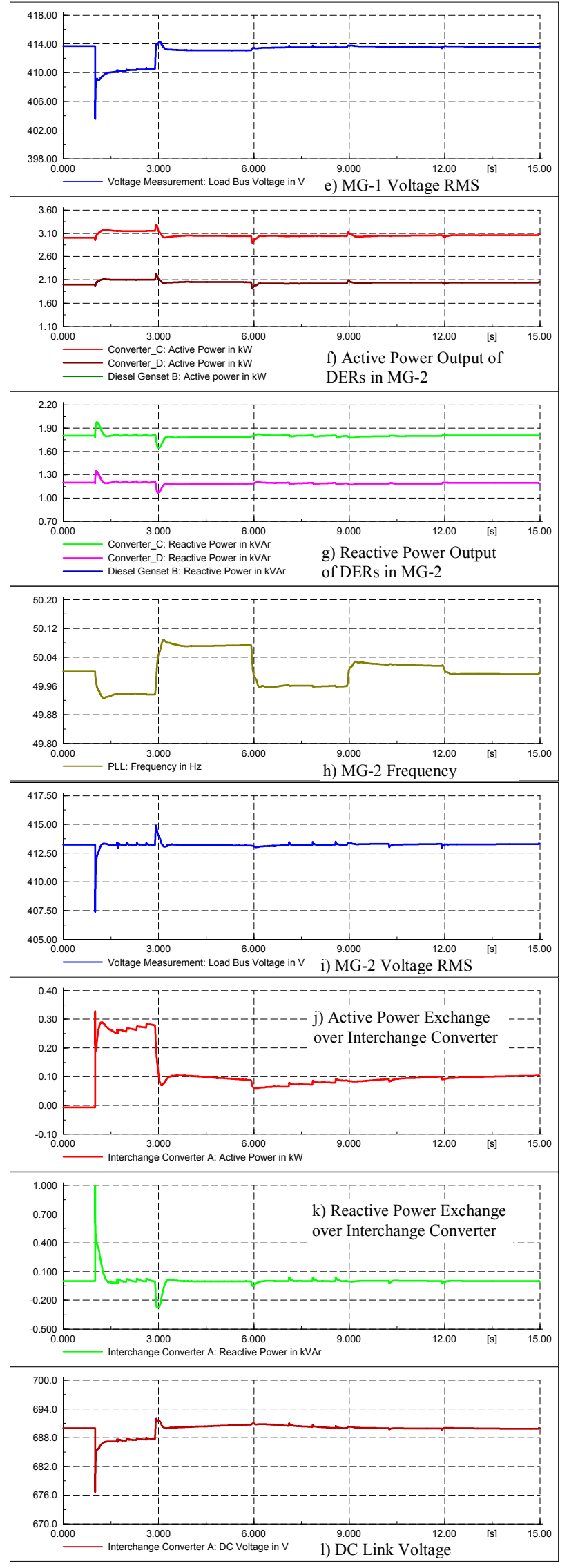

Fig. 4. Case 1 simulation results (continued). 
Let us assume in the steady state, MG-1 has a load demand of $16 \mathrm{~kW}$ while MG-2 has a load demand of $5 \mathrm{~kW}$, both at $\mathrm{pf}=$ 0.85 lagging. It is also assumed that, based on the secondary controller, in each MG this power is shared with a ratio of $60 / 40 \%$ between the two converter-interfaced DERs while the diesel generator is assumed to be running at no-load condition.

At $t=1 \mathrm{~s}$, a $10 \mathrm{~kW}$ load demand (with $\mathrm{pf}=0.85$ lagging) is added to MG-1 (Fig. 5a), bringing the total load demand to $26 \mathrm{~kW}$ and thereby overloading MG-1. The results of the simulation are shown in Fig. 5 for MG-1.

As expected, it can be seen that the DERs in MG-1 reach their active and reactive power limits (i.e. $10 \mathrm{kVA}$ ) quickly after the load change (Fig. 5b-c). As a result of the overloading condition, the frequency and voltage decline rapidly to below the acceptable limits of $49 \mathrm{~Hz}$ and 0.9 pu voltage within 1.3 seconds (Fig. 5d-e).

The diesel generator also reaches its active power limit soon after the load change (Fig. 5b), but the overloading condition causes the rotational speed of the shaft to slow down and consequently a decline in frequency.

Under-frequency or under-voltage protection relays are not modelled in this research, but such an overloading condition would result in load shedding through the activation of underfrequency or under-voltage relays or in the worst case, a system blackout.

\section{Case 3: MG Overload Operation with Interchange Converter}

The interchange converter is intended to provide frequency support by facilitating active power exchange between $M G$ clusters (in the event of overloading within one MG cluster). In Case 3, the interchange converter is put in service and the overload simulation in Case 2 is repeated.

Let us assume the steady state conditions same as Case 2 . At $t=1 \mathrm{~s}$, a $10 \mathrm{~kW}$ load demand is added to MG-1. The results of the simulation are shown in Fig. 6.

For the power demand variation in MG-1 (Fig. 6a), similar to Case 2, the DERs in MG-1 (converter-A and B) quickly reach their active and reactive power limits of $10 \mathrm{kVA}$ (Fig. $6 \mathrm{~b}-\mathrm{c}$ ) and the frequency and voltage rms in MG-1 drops (Fig. 6d-e). However in this case, the DERs (converter-C and D) increase their output power with the pre-assigned ratio of $60 / 40 \%$ (Fig. 6f-g). Hence, after this increase, the frequency of MG-2 is decreased from $50 \mathrm{~Hz}$ to $49.2 \mathrm{~Hz}$ (Fig. 6h) and the voltage rms drops from 1.00 pu to 0.96 pu (Fig. 6i), after the increase in the output power of these two converters.

The diesel generator in MG-1 increases its active power output in response to the load change and quickly reaches its limits. However, the active power support from MG-2 allows the diesel generator to reduce its power output, finally settling at $1.6 \mathrm{~kW}$ through droop control action (Fig. 6b). There is negligible response from the diesel generator in MG-2.

Hence, based on the frequency drop in MG-1, the interchange converter acts to facilitate active power transfer of approximately $5 \mathrm{~kW}$ and zero kVAr from MG-2 to MG-1 (Fig. 6j-k), allowing the frequency and voltage in MG-1 to recover to nominal values.

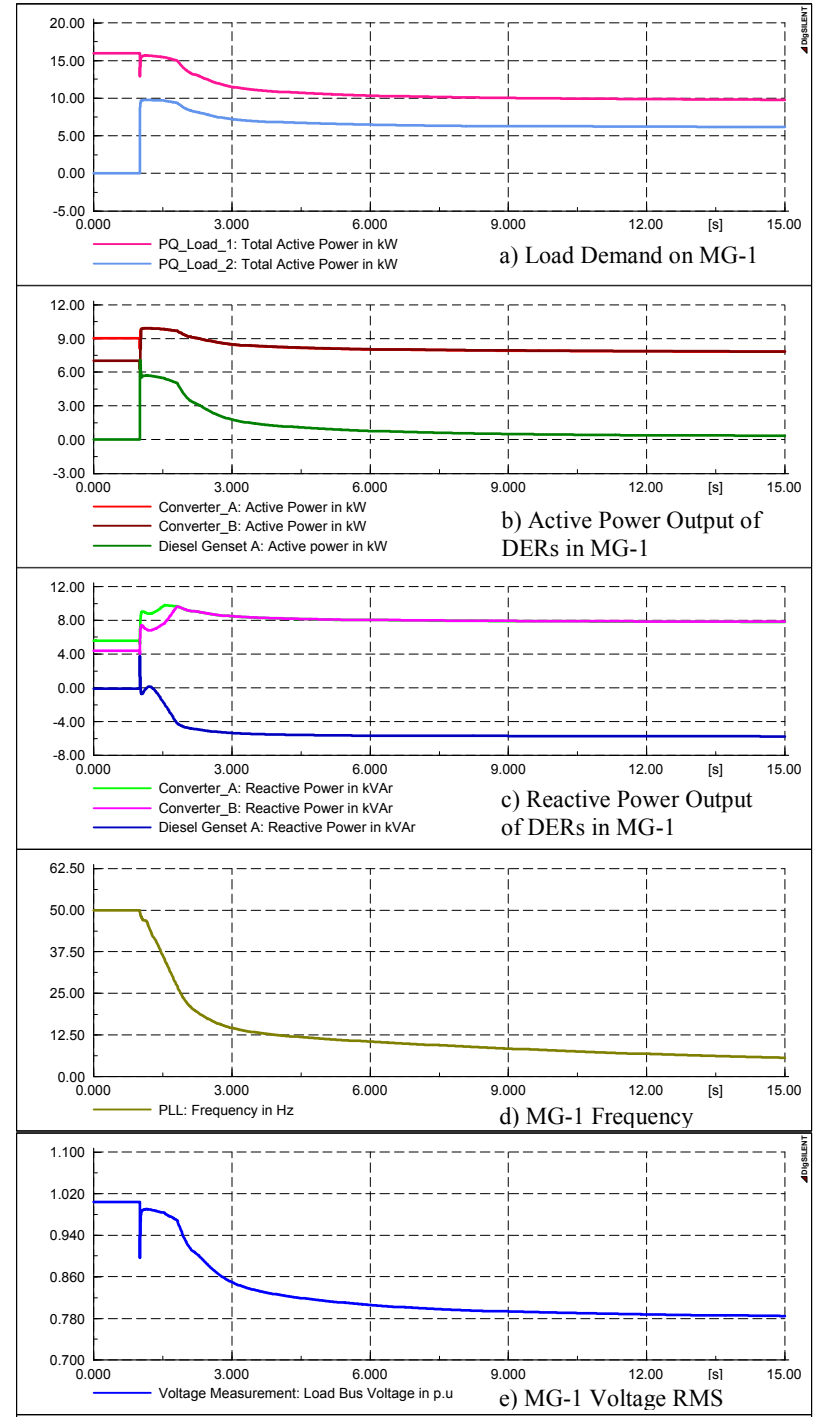

Fig. 5. Case 2 simulation results for MG-1.

It is to be noted that the two MGs are decoupled from each other during the overloading event. As an example, a large peak frequency deviations of 35\% in MG-1 (Fig. 6d) results in a peak frequency deviations of just $2.4 \%$ in MG-2 (Fig. 6h).

\section{CONCLUSION}

The concept of interconnecting two neighbouring autonomous MGs, used as the power supply units in remote areas, is discussed as a method to prevent load shedding. The two MGs are interconnected together through a back-to-back interchange converter arrangement. This configuration provides dynamic frequency support in the MGs in the case of overloading or deficiency in power generation in either of the MGs. Through the simulation studies, it is demonstrated that the interchange converter successfully facilitates active power flow from one MG to the other MG based on the frequency deviation. As a future study, the operation of more than two MGs, interconnected through a DC link can be investigated. 

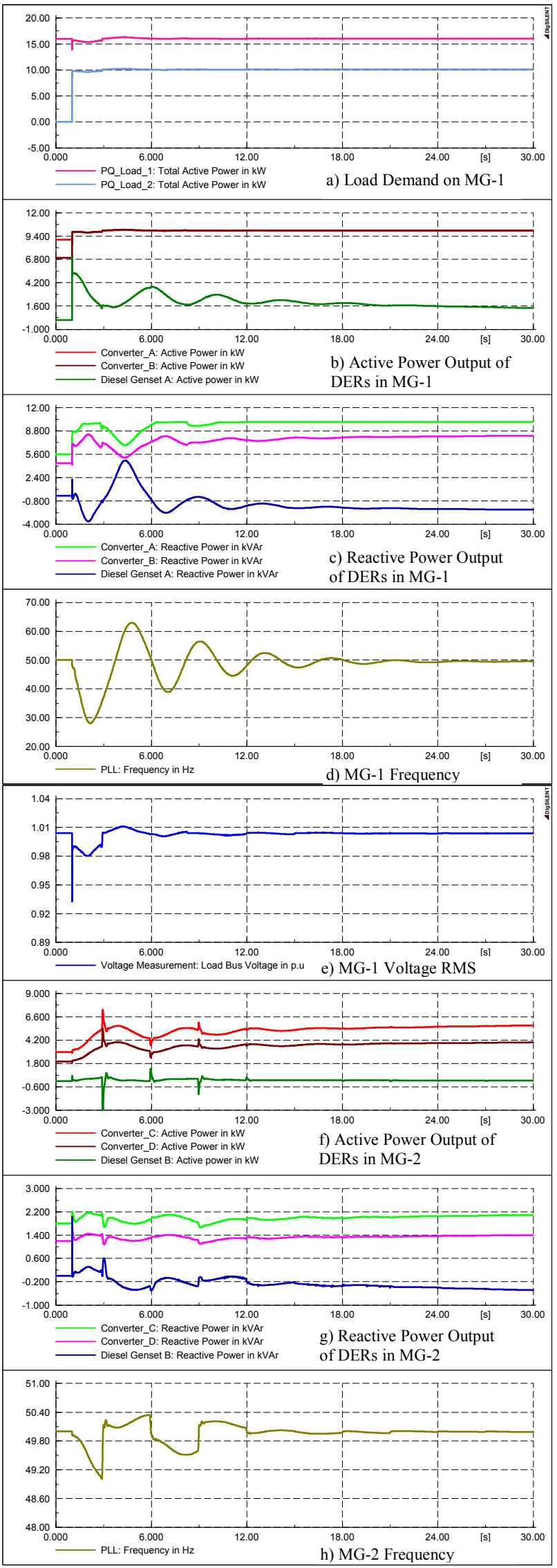

Fig. 6. Case 3 simulation results.

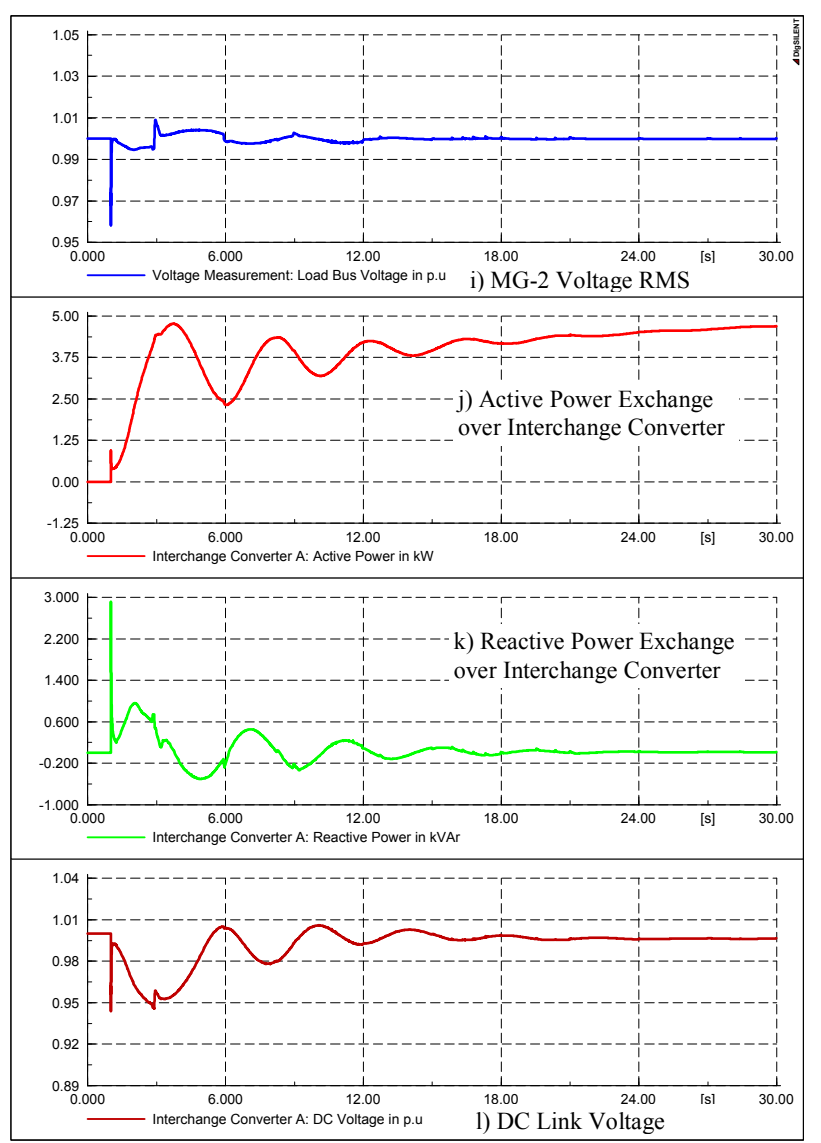

Fig. 6. Case 3 simulation results (continued).

\section{REFERENCES}

[1] R.H. Lasseter, "Microgrids and distributed generation", Journal of Energy Engineering, vol. 133, no. 3, pp. 144-149, Sept. 2007.

[2] H. Zeineldin, E.F. El-Saadany and M.M.A. Salama, "Intentional islanding of distributed generation," IEEE Power and Energy Society General Meeting, vol. 2, pp.1496-1502, 2005.

[3] N. Hatziargyriou, H. Asano, R. Iravani and C. Marnay, "Microgrids," IEEE Power Energy Magazine, vol. 5, no. 4, pp. 78-94, Jul./Aug. 2007.

[4] G. Pepermans, J. Driesen, D. Haeseldonckx, et al. "Distributed generation: definition, benefits and issues," Energy Policy, vol. 33, Issue 6, pp. 787-798, April, 2005.

[5] R. Majumder, B. Chaudhuri, A. Ghosh, et al. "Improvement of stability and load sharing in an autonomous microgrid using supplementary droop control loop," IEEE Trans. on Power Systems, vol. 25, no. 2, pp. 796-808, May 2010.

[6] F. Shahnia, R.P.S. Chandrasena, S. Rajakaruna and A. Ghosh, "Primary control level of parallel distributed energy resources converters in system of multiple interconnected autonomous microgrids within selfhealing networks," IET Generation, Transmission \& Distribution, vol. 8, no. 2, pp. 203-222, Feb. 2014.

[7] Siemens PTI, "Block Oriented Simulation Language (BOSL) Standard Controllers", 2008.

[8] IEEE recommended practice for excitation system models for power system stability studies, IEEE Std. 421.5, 2005.

[9] J.M. Guerrero, J.C. Vasquez, J. Matas, et al. "Hierarchical control of droop-controlled AC and DC microgrids-A general approach toward standardization," IEEE Trans. on Industrial Electronics, vol. 58, no. 1, pp. 158-172, Jan. 2011.

[10] K. De Brabandere, K. Vanthournout, J. Driesen, et al. "Control of microgrids", IEEE Power and Energy General Meeting, pp. 1-7, 2007.

[11] R. Teodorescu and F. Blaabjerg, "Flexible control of small wind turbines with grid failure detection operating standalone and gridconnected mode", IEEE Trans. on Power Electronics, vol. 19, no. 5, pp. 1323-1332, Sept. 2004. 